\title{
A Note on Bounds of Multiple Characteristic Roots of a Matrix
}

\author{
P. Stein ${ }^{2}$ \\ If $A=\left(a_{i s}\right)$ is an $n \times n$ matrix, and if $C_{i}$ are the circles, center $a_{i i}$ and radii $\sum_{\substack{s=1 \\ s \neq i}}^{n}\left|a_{i s}\right|$, and if
}

$\lambda$ is a characteristic root with $m$ independent characteristic vectors, Olga Taussky proved the following two results:

(1) If $\lambda$ lies outside all but one circle $C_{i}$, then $m$ cannot be greater than 1.

(2) If $m=n-1$, then $\lambda$ is an inner or boundary point of at least $m$ circles $C_{i}$.

In this note the gap between these two results is closed, and it is shown that $\lambda$ lies in at least $m$ circles $C_{i}$, for all finite values of $m$ and $n, m \leqq n$.

If $\boldsymbol{A}=\left(a_{i j}\right)$ is an $n \times n$ matrix and if $C_{i}$ are the circles with centres $a_{i i}$ and radii $\sum_{\substack{s=1 \\ s \neq i}}^{n}\left|a_{i s}\right|$, Olga Taussky ${ }^{3}$ proves these two theorems.

Theorem A. A characteristic root $\lambda$, which is an inner or boundary point of only one $C_{i}$, cannot have two independent characteristic vectors corresponding to it.

Theorem B. If $A$ has a characteristic root $\lambda$ of multiplicity $n-1$, with $n-1$ independent characteristic vectors, the $\lambda$ lies in at least $n-1$ circles $C_{i}$.

In this note it is proved that

Theorem C. If $\lambda$ is a characteristic root of $A$ with $m \leq n$ independent characteristic vectors corresponding to it, then $\lambda$ lies in at least $m$ circles $C_{i}$.

Theorem $\mathrm{C}$ is a generalization of both Theorems $\mathrm{A}$ and $\mathrm{B}$ and closes the gap between them.

Theorem $\mathrm{C}$ contains the following generalization of a well-known theorem about determinants (for definitions and references, see, O. Taussky, A recurring theorem on determinants, Am. Math. Monthly 56, $672(1949))$.

Theorem $D .^{4}$ Let $A$ be a matrix that cannot be transformed to the form $\left(\begin{array}{ll}P & U \\ O & Q\end{array}\right)$ by the same permutation of the rows and columns, where $O$ consists of zeros, and $P$ and $Q$ are square matrices. Let further $\left|a_{i i}\right| \neq \sum_{\substack{s=1 \\ s \neq i}}^{n}\left|a_{i s}\right|$ for at least one value of $i$. If the rank of the matrix $A$ is $n-m$, where $O \leq m \leq n$ then there must be at least $m$ values of $i$ for which the inequalities

$$
\left|a_{i i}\right|<\sum_{\substack{s=1 \\ s \neq i}}^{n}\left|a_{i s}\right|
$$

hold.

\footnotetext{
1 This work was performed on a National Bureau of Standards contract with the University of California at Los Angeles and was sponsored (in part) by the Office of Naval Research.

2 University of Natal, Durban, South Africa, and University of California at

$3 \mathrm{O}$. Taussky, Bounds for characteristic roots of matrices II, J. Research
Los NBS 46, 124 (1951) R P2184.

4 This was pointed out by $\mathrm{O}$. Taussky.
}

We require this lemma:

Lemma. If $X_{i}, i=1,2, \ldots, m$, are $m$ independent vectors with components $x_{i s}, s=1,2, \ldots ., n, n \geq m$, we may construct a set of $m$ independent vectors $Y_{i}$, with components $y_{i s}$, which are linear combinations of the vectors $X_{i}$ and which have the property that we may select components of maxima moduli corresponding to each $Y_{i}$, so that no two such selected components have the same subscripts.

We may suppose $m \geq 2$.

We choose $Y_{1}=X_{1}$. Let $y_{1 s_{1}}=x_{1 s_{1}}$ be a component of maximum modulus of $Y_{1}$. Choose $\alpha_{1}$ and $\alpha_{2}$ so that

$$
\alpha_{1} y_{1 s_{1}}+\alpha_{2} x_{2 s_{1}}=0
$$

and

$$
Y_{2}=\alpha_{1} Y_{1}+\alpha_{2} X_{2}
$$

Since $y_{i s_{1}} \neq 0, \alpha_{2} \neq 0$, and so since $X_{1}$ and $X_{2}$ are linearly independent, $Y_{2} \neq 0$. Let $y_{2 s_{2}}$ be a component of maximum modulus of $Y_{2}$. By (1) and (2) $y_{2 s_{1}}=0$, hence $s_{2} \neq s_{1}$. Further, $Y_{1}$ and $Y_{2}$ are linear combinations of the vectors $X_{1}$ and $X_{2}$ and are independent. The construction is thus complete for two independent vectors $Y_{i}$. If $m \geq 3$, we choose three numbers $\beta_{1}, \beta_{2}, \beta_{3}$ so that

$$
\begin{aligned}
& \beta_{1} y_{1 s_{1}}+\beta_{2} y_{2 s_{1}}+\beta_{3} x_{3 s_{1}}=0 \\
& \beta_{1} y_{1 s_{2}}+\beta_{2} y_{2 s_{2}}+\beta_{3} x_{3 s_{2}}=0
\end{aligned}
$$

and

$$
Y_{3}=\beta_{1} Y_{1}+\beta_{2} Y_{2}+\beta_{2} X_{3} \text {. }
$$

Since

$$
\left|\begin{array}{ll}
y_{1 s_{1}} & y_{2 s_{1}} \\
y_{1 s_{2}} & y_{2 s_{2}}
\end{array}\right|=y_{1 s_{1}} y_{2 s_{2}} \neq 0
$$

$\beta_{3} \neq 0$, and $Y_{3} \neq 0$. The argument used above may now be repeated to show that if $y_{3 s_{3}}$ is a component of maximum modulus of $Y_{3}$, then $s_{3} \neq s_{1}, s_{3} \neq s_{2}$. Further, $Y_{3}$ has the other properties required of $Y_{i}$. 
This would complete the construction for three vectors $X_{i}$.

If $m \geq 4$, the other properties may be continued step by step till all the independent vectors $Y_{i}$ are exhausted. This completes the proof of the Lemma. To complete the proof of Theorem $\mathrm{C}$, given the set of $m$ independent characteristic vectors $X_{i}$, corresponding to the characteristic root $\lambda$, we construct the set $Y_{i}$ of the Lemma. Since $Y_{i}$ are linear combinations of $X_{i}$, they are also characteristic vectors corresponding to the characteristic root $\lambda$. Hence we have the system of equations

$$
\sum_{s=1}^{n} a_{t s} y_{i s}=\lambda y_{i t}, i=1,2, \ldots, m ; t=1,2, \ldots, n .
$$

In particular we have

$$
\sum_{s=1}^{n} a_{s_{r}} y_{i s}=\lambda y_{i s_{\imath}}
$$

and so

$$
\left(\lambda-a_{s_{i} s_{i}}\right) y_{i s_{i}}=\sum_{\substack{s=1 \\ s \neq s_{i}}}^{n} a_{s_{i}} y_{i s}
$$

Dividing through by $y_{i s_{i}}$, and taking the moduli of the two sides, since $\left|y_{i s_{i}}\right| \geq\left|y_{i s}\right|, s=1,2, \ldots, n$ we get that $\lambda$ lies in the circle $C_{s_{i}}$.

Since $s_{i} \neq \mathrm{s}_{j}, \quad i \neq j$, we conclude that $\lambda$ lies in $m$ different circles $C_{i}$. This concludes the proof of the theorem.

Los Angeles, October 18, 1951. 